\title{
Metabolism of tocotrienols in animals and synergistic inhibitory actions of tocotrienols with atorvastatin in cancer cells
}

\author{
Zhihong Yang • Mao-Jung Lee $\cdot$ Yang Zhao • \\ Chung S. Yang
}

Received: 18 April 2011/Accepted: 22 April 2011/Published online: 18 May 2011

(C) Springer-Verlag 2011

\begin{abstract}
Tocotrienols (T3s), members of the vitamin E family, exhibit potent anti-cancer, anti-oxidative, antiinflammatory, and some other biological activities. To better understand the bioavailability and metabolism of $\mathrm{T} 3 \mathrm{~s}, \mathrm{~T} 3 \mathrm{~s}$ and their metabolites were identified in urine and fecal samples from mice on diet supplemented with mixed T3s using HPLC/electrochemical detection and liquid chromatography electrospray ionisation mass spectrometry (LC-ESI-MS). Whereas the short-chain metabolites carboxyethyl hydroxychromans (CEHCs) and carboxymethylbutyl hydroxychromans (CMBHCs) were the major metabolites of T3s, several new metabolites with double bonds were also identified. Similar to tocopherols, the majority of T3 metabolites were excreted as sulfate/ glucuronide conjugates in mouse urine. The distribution of $\gamma$ - and $\delta$-T3 and $\gamma$-T3 metabolites were also determined in different organs as well as in urine and fecal samples from mice on diets supplemented with corresponding T3s. The synergistic anti-cancer actions of $\gamma$-T3 and atorvastatin (ATST) were studied in HT29 and HCT116 colon cancer cell lines. The combination greatly potentiated the ability
\end{abstract}

This paper is part of a Special Issue entitled "Tocotrienols in health and disease" (Guest editors: Bharat B. Aggarwal and Kalaniti Nesaretnam)".

Z. Yang · M.-J. Lee · Y. Zhao · C. S. Yang $(\varangle)$

Susan Lehman Cullman Laboratory for Cancer Research, Department of Chemical Biology and Center for Cancer Prevention Research, Ernest Mario School of Pharmacy, Rutgers, The State University of New Jersey,

164 Frelinghuysen Road, Piscataway, NJ 08854, USA

e-mail: csyang@rci.rutgers.edu

C. S. Yang

Cancer Institute of New Jersey,

195 Little Albany Street, New Brunswick, NJ 08901, USA of each individual agent to inhibit cancer cell growth and to induce cell cycle arrest and apoptosis. The triple combination of $\gamma$-T3, ATST, and celecoxib exhibited synergistic actions when compared with any double combination plus the third agent. Mechanistic studies revealed that the synergistic actions of $\gamma$-T3 and ATST could be attributed to their mediation of 3-hydroxy-3-methyl-glutaryl-CoA reductase, and the subsequent inhibition of protein geranylgeranylation. It remains to be determined whether such a synergy occurs in vivo.

Keywords Tocotrienol - Metabolites · HPLC . Atorvastatin · Synergy

\begin{tabular}{ll}
\multicolumn{2}{l}{ Abbreviation } \\
T3 & Tocotrienol \\
CEHC & Carboxyethyl hydroxychromans \\
CMBHC & Carboxymethylbutyl hydroxychromans \\
HMGCR & 3-hydroxy-3-methylglutaryl coenzyme \\
& A reductase \\
MTT & 3-(4, 5-dimethylthiazol-2-yl)-2, \\
& 5-diphenyltetrazolium bromide
\end{tabular}

\section{Introduction}

The vitamin E family is composed of tocopherols and tocotrienols (T3s). The difference between tocopherols and $\mathrm{T} 3 \mathrm{~s}$ is that tocopherols contain a 16-carbon saturated phytyl side chain, whereas $\mathrm{T} 3 \mathrm{~s}$ possess an unsaturated isoprenoid side chain. Based on the number and location of the methyl groups on their chromanol rings, T3s and tocopherols exist in $\alpha$-, $\beta$-, $\gamma$-, and $\delta$-forms (Fig. 1). Emerging evidence suggests that T3s exhibit more potent anti-cancer 
Fig. 1 The structures of tocopherols and tocotrienols<smiles>[R6]c1c([B])c2c(c(Br)c1O)CCC(C)(CCCC(C)CCCC(C)CCCC(C)C)O2</smiles><smiles>[2H]c1c([2H])c2c(c(Br)c1O)CCC(C)(CC/C=C(\C)CC/C=C(\C)CCC=C(C)C)O2</smiles>

Tocotrienol

$\alpha$-form: $\mathrm{R}_{1}=\mathrm{R}_{3}=\mathrm{R}_{3}=\mathrm{CH}_{3}$

$\beta$-form: $\mathrm{R}_{1}=\mathrm{R}_{3}=\mathrm{CH}_{3}, \mathrm{R}_{2}=\mathrm{H}$

$\gamma$-form: $\mathrm{R}_{2}=\mathrm{R}_{3}=\mathrm{CH}_{3}, \mathrm{R}_{1}=\mathrm{H}$

$\delta$-form: $\mathrm{R}_{3}=\mathrm{CH}_{3}, \mathrm{R}_{1}=\mathrm{R}_{2}=\mathrm{H}$

effects than tocopherols. In this article, we review our works on the tissue distribution and metabolism of T3s and their metabolites as well as the synergistic action of $\gamma$-T3 with atorvastatin and celecoxib. Some recent progress in the various actions of $\mathrm{T} 3 \mathrm{~s}$ is also summarized.

\section{The metabolism of tocotrienols and the tissue distribution of tocotrienols and their metabolites}

Recent studies show that the metabolites of vitamin E may also contribute to the biological effects of vitamin E (Jiang et al. 2008; Birringer et al. 2010). Therefore, it is important to understand the metabolism of vitamin E. Although the metabolism of tocopherols has been extensively investigated, studies on the metabolism of $\mathrm{T} 3 \mathrm{~s}$ are limited. It is believed that, similar to tocopherols, T3s are metabolized through the oxidative degradation of their side chains. The degradation involves cytochrome P-450-catalyzed $\omega$-hydroxylation and then oxidation to $\omega$-carboxylic acid, followed by five cycles of $\beta$-oxidation to remove a two carbon moiety each cycle from the side chain (Birringer et al. 2002). The final products of the degradation are carboxyethyl hydroxychromans (CEHCs) and their precursors, carboxymethylbutyl hydroxychromans (CMBHCs).

Our group established a sensitive HPLC/electrochemical detection (ECD) method to analyze T3s and their metabolites. We first used this sensitive HPLC/ECD coupled with LC-MS to identify and characterize different side chain metabolites of T3s in mouse fecal and urine samples (Zhao et al. 2010). In fecal samples from the mice fed with diets supplemented with mixed T3s $(20.2 \% \alpha$-T3, $4.0 \% \quad \beta$-T3, $16.1 \% \gamma$-T3, 9.9\% $\delta$-T3, $14.8 \% \alpha$-tocopherol, and $3.1 \%$ $\gamma$-tocopherol), $\delta$-, $\gamma$-, and $\alpha$-CEHCs and CMBHCs, as well as some new metabolites, were observed in HPLC chromatogram. A new metabolite was identified as $\gamma$-carboxymethylbutenyl hydroxychroman (CMBenHC) (Zhao et al. 2010). It is worth noting that more short-chain metabolites of $\mathrm{T} 3 \mathrm{~s}$ were present than long-chain metabolites. Further analysis of the fecal samples by LC-MS/MS revealed that $\mathrm{T} 3$ metabolites included CEHCs, CMBHCs, carboxymethylhexenyl hydroxychromans (CMHenHCs), carboxydimethyloctenyl hydroxychromans (CDMOenHCs), and carboxydimethyldecadienyl hydroxychromans $\left(\mathrm{CDMD}(\mathrm{en})_{2} \mathrm{HCs}\right)$. These metabolites are similar to those reported in HepG2 cells (Birringer et al. 2002). Importantly, we also identified three additional groups of T3 metabolites: major peaks of CMBenHCs with one double bond in the side chain, minor peaks of carboxydimethyloctadienyl hydroxychromans $\left(\mathrm{CDMO}(\mathrm{en})_{2} \mathrm{HCs}\right)$ with two double bonds, and carboxyl tocotrienols (Zhao et al. 2010). Based on these results, we proposed the degradation pathway of $\mathrm{T} 3 \mathrm{~s}$ as shown in Fig. 2. In the urine samples from mice fed diet supplemented with mixed T3s, short-chain metabolites including $\mathrm{CEHCs}$, CMBHCs, and CMHenHCs were detected, whereas the parental compounds of T3s were not detected.

Using the opportunity of our studies on the anti-cancer activities of $\mathrm{T} 3 \mathrm{~s}$ and tocopherols in mouse tumor xenograft models, we determined the levels of T3s and compared their levels with those of corresponding tocopherols. The level of $\gamma$-T3 metabolites was also studied in several mouse 
Fig. 2 A proposed tocotrienol degradation pathway
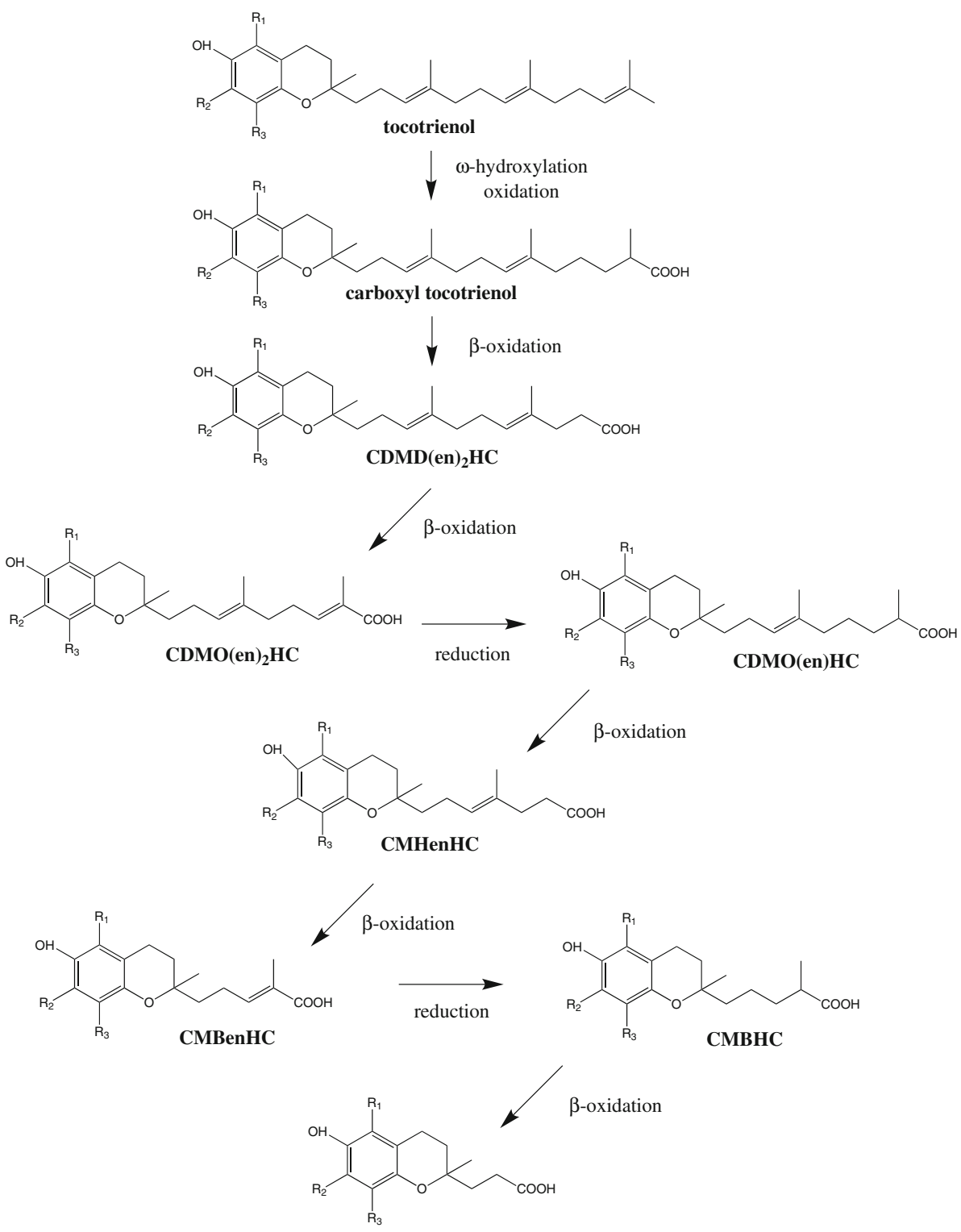

CEHC

tissues. $\mathrm{NCr} \mathrm{Nu} / \mathrm{Nu}$ mice were supplemented with diets containing 0.1 and $0.3 \% \gamma$-tocopherol $(\gamma$-T), 0.1 and $0.3 \%$ $\delta$-T, $0.1 \% \gamma$-T3, and $0.1 \% \delta$-T3 for 1 week, and $1 \times 10^{6}$ H1299 lung cancer cells were then inoculated to both flank sides of each mouse. The mice continued to be fed on the same diet for another 6 weeks before they were killed. Supplementation of T3s increased the level of corresponding T3s in mouse serum and all the organs examined. The levels of $\mathrm{T} 3 \mathrm{~s}$ were relatively low in the liver $(0.5 \mu \mathrm{mol} / \mathrm{kg})$ and high in the spleen, kidney, small intestine, and colon $(>10 \mu \mathrm{mol} / \mathrm{kg})$. The levels of T3s in the xenograft tumor and lung were in the range of $1-10 \mu \mathrm{mol} / \mathrm{kg}$. The plasma levels of $\mathrm{T} 3 \mathrm{~s} \quad(1.63 \pm 0.61 \mu \mathrm{M}$ and $0.59 \pm 0.08 \mu \mathrm{M}$ for $\gamma$-T3 and $\delta$-T3, respectively) were lower than those of the corresponding tocopherols
$(4.75 \pm 1.29 \mu \mathrm{M}$ and $1.60 \pm 0.21 \mu \mathrm{M}$ for $\gamma$-T and $\delta$-T, respectively) from mice fed the diets supplemented with the same concentration of tocopherols. In contrast, the T3s levels in the spleen, kidney, small intestine, and colon were higher than those of corresponding tocopherols. The levels of T3s and tocopherols were similar in the lung, liver, and xenograft tumors (data not shown).

To investigate the tissue distribution of T3 metabolites, athymic nude mice bearing A549 lung cancer cell xenograft tumors were fed AIN76m control diet (modified from AIN76A rodent diet by using stripped corn oil, which contains no tocopherols, and adding $32 \mathrm{mg}$ tocopheryl acetate per $\mathrm{kg}$ diet to meet the nutritional requirement) or AIN76 $m$ diet supplemented with $0.05 \% \gamma$-T3 for 2 weeks. $\gamma$-T3 was detected in the serum samples as well as in 
several organs (lung, liver, spleen, and colon) from the mice in the $\gamma$-T3 group, but not in the control diet group. Several putative medium and long-chain metabolites were also detected in the $\gamma$-T3 group, particularly in colon samples. It is worth noting that $\gamma$-tocopherol $(\gamma$-T) was detected in the lung, liver, and colon samples from both groups. However, it is likely that $\gamma$-T only contributed a minor portion to the total levels of short-chain metabolites as compared to $\gamma$-T3 because in the control diet group, $\gamma$-CEHC and $\gamma$-CMBHC were either not detectable or at levels much lower than in the $\gamma$-T3 group, although the levels of $\gamma$-T in both groups were comparable (data not shown). The representative chromatograms of $\gamma$-T3 and its short-chain metabolites in the serum samples are shown in Fig. 3a and b, respectively, and the levels of $\gamma-\mathrm{T} 3$ and its short metabolites in the serum, lung, liver, spleen, colon, and urine samples were summarized in Table 1. Hydrolysis of the metabolites by glucuronidase and sulfatase in the urine samples dramatically increased the levels of $\gamma$-CEHC and $\gamma$-CMBHC in comparison to those in unhydrolyzed samples, suggesting that $\gamma$-CEHC and $\gamma$-CMBHC were excreted in mouse urine as glucuronidated or sulfated forms (Table 1). This conclusion is similar to the detection of sulfated/glucuronidated T3 long-chain metabolites with 9-, 11-, and 13-carbon side chains and the result that most of the $\gamma$-CEHC was in conjugated forms in plasma samples from a study of rats gavaged by a single dose of $\gamma$-T3 (Freiser and Jiang 2009). Consistent with previous experiments, the $\gamma$-T3 level in the liver was the lowest among the organs examined. In the serum and lung samples, $\gamma$-T3 levels were higher than its short-chain metabolites whereas in the liver, the level of $\gamma$-T3 was much lower than the metabolites. In the spleen, $\gamma$-T3 level is higher than $\gamma$-CMBHC, but lower than $\gamma$-CEHC (Table 1). The $\gamma$-T3 metabolites were high in the colon and urine, suggesting that $\gamma$-T3 is metabolized in the liver, and the metabolites are excreted from urine and bile. It is worth noting that $\gamma$-CEHC is the major metabolite in the urine, whereas the level of $\gamma$-CMBHC is higher than $\gamma$-CEHC in the liver and colon.

In summary, our studies established the methodology to measure the complete profile of T3 metabolites in mice. We obtained information about the distribution of $\gamma$ - and $\delta$-T3 and $\gamma$-T3 metabolites in different mouse tissues. Our data will be very useful in future studies on the relationship between T3 metabolites and their biological functions.

\section{Anti-cancer effects of tocotrienols}

The anti-cancer effects are the most studied functions of T3s. T3s prevent proliferation and induce apoptosis in various cancer cell lines by affecting MAPK, PI3K/Akt,
EGFR, NF- $\kappa$ B, and PPAR-related pathways (Sun et al. 2008; Samant and Sylvester 2006; Shah and Sylvester 2005; Yap et al. 2008; Fang et al. 2010). It is worth noting that $\mathrm{T} 3 \mathrm{~s}$ may also induce cancer cell apoptosis independent of mitochondria stress-mediated and death receptor-related apoptosis pathways (Shah and Sylvester 2004, 2005). T3s also induce autophagy, a key cellular process related to cell metabolism and cancer, in activated rat pancreatic stellate cells (Rickmann et al. 2007). Autophagy may also contribute to the synergistic cardioprotection in rats conferred by the combination treatment with resveratrol and $\gamma$-T3 (Lekli et al. 2009). However, the T3-induced autophagy is poorly understood and its roles in anti-cancer actions still need to be further elucidated. T3s also inhibit the levels of cell adhesion proteins and block tumor angiogenesis (Miyazawa et al. 2008), which may also contribute to their anti-cancer activities.

Despite the large number of the anti-cancer studies of $\mathrm{T} 3 \mathrm{~s}$, many of them were still limited to phenomenon observations on the change of certain signal molecules without an in-depth insight about the mechanisms. Aggarwal and coauthors reported that $\gamma-\mathrm{T} 3$ blocked NF- $\kappa \mathrm{B}$ activation, TNF-induced phosphorylation, and $\mathrm{I} \kappa \mathrm{B} \alpha$ degradation (Ahn et al. 2007). They also demonstrated that $\gamma$-T3 inhibited the activation of STAT3, possibly through the induction of protein tyrosine phosphatase SHP-1 (Kannappan et al. 2010). The levels of some NF- $\kappa$ B- and STAT3-mediated gene products in cancer cells associated with anti-apoptosis, proliferation, invasion, and angiogenesis were down-regulated by $\gamma$-T3. Their studies provided more insightful mechanisms about the actions of $\gamma$-T3. However, none of the proteins described above were proposed as a direct target of $\gamma$-T3. A recent study with in silico simulation and in vitro binding assay suggested a high affinity binding between T3s and estrogen receptor- $\beta$ $(\operatorname{ER} \beta)$, but not $\operatorname{ER} \alpha$ (Comitato et al. 2009). It was demonstrated that $\mathrm{T} 3 \mathrm{~s}$ could promote the translocation of $\mathrm{ER} \beta$ into the nucleus and activate some estrogen-responsive genes in breast cancer cell lines (Comitato et al. 2009, 2010). In addition to the effects of T3s on signaling pathways in cancer cell lines, T3 may also target cancer stem cell-like population in that $\gamma$-T3 down-regulated the level of prostate cancer stem cell markers (CD133/CD44) and reduced the spheroid formation of prostate cancer cells (Luk et al. 2010).

T3s have been shown to reduce the level of 3-hydroxy3-methylglutaryl coenzyme A reductase (HMGCR), the rate-limiting enzyme in cholesterol biosynthesis, in hepatic HepG2 cells at post-transcriptional level (Parker et al. 1993). Indeed, $\delta$ - and $\gamma$-T3 promoted endoplasmic reticulum protein Insig (insulin induced gene)-induced ubiquitination and degradation of HMGCR (Song and DeBose-Boyd 2006). These results suggest the role of T3s 
Fig. 3 Chromatograms of $\gamma$-tocotrienol (a) and its shortchain metabolites (b) in mouse serum samples
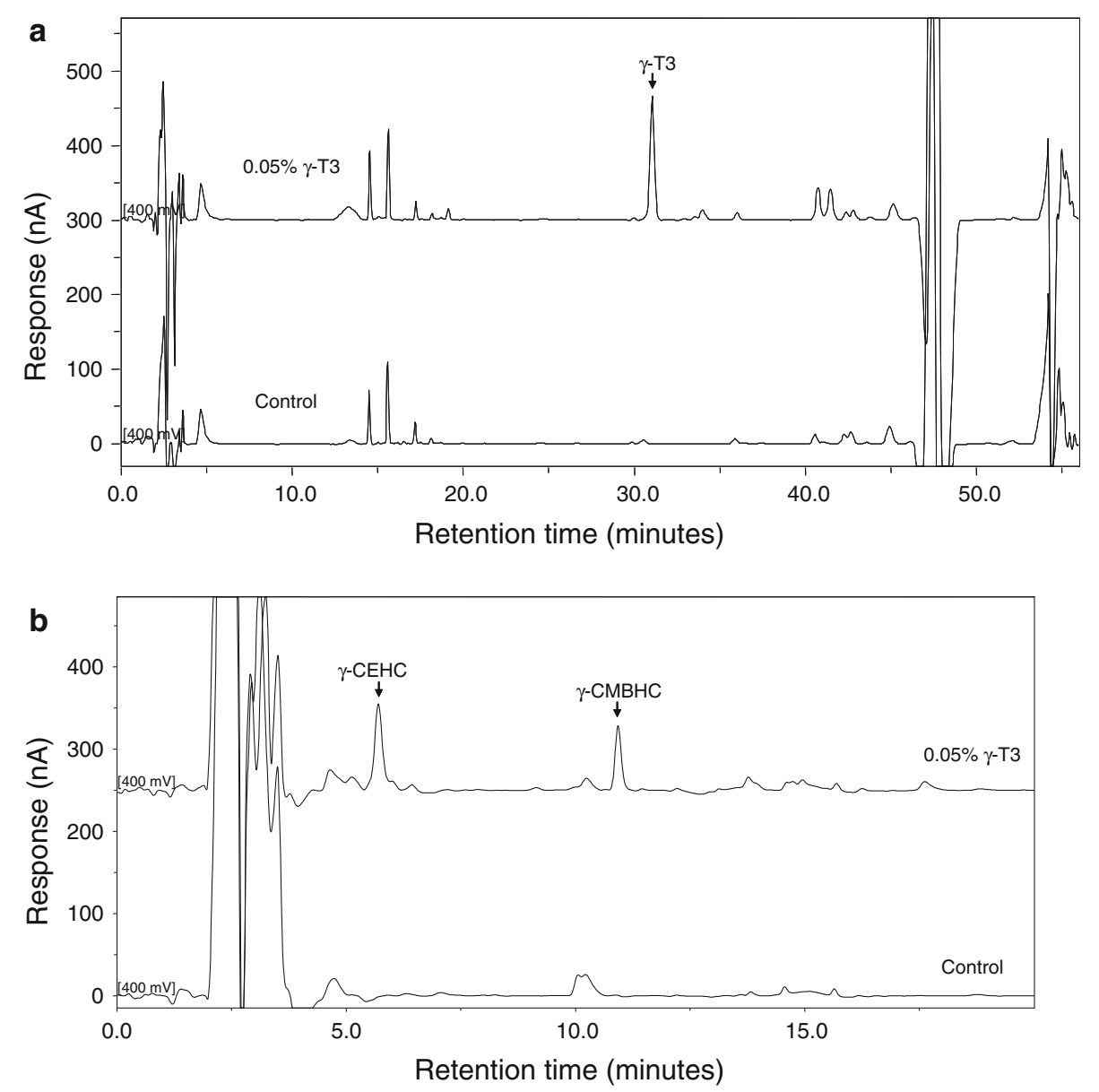

Table 1 The levels of $\gamma$-T3 and its short-chain metabolites ( $\gamma$-CEHC and $\gamma$-CMBHC) in serum, lung, liver, spleen, colon, and urine samples from mice treated with $\gamma$-T3

\begin{tabular}{llrr}
\hline Sample name & \multicolumn{1}{c}{ ${ }^{\mathrm{a}}$-CEHC } & \multicolumn{1}{c}{$\gamma$-CMBHC } & $\gamma$-T3 \\
\hline Serum $(\mu \mathrm{M})$ & $0.09 \pm 0.03$ & $0.12 \pm 0.04$ & $0.39 \pm 0.07$ \\
Lung $(\mu \mathrm{mol} / \mathrm{kg})$ & $0.06 \pm 0.02$ & $0.18 \pm 0.02$ & $0.92 \pm 0.14$ \\
Liver $(\mu \mathrm{mol} / \mathrm{kg})$ & $2.75 \pm 0.50$ & $15.32 \pm 1.24$ & $0.10 \pm 0.01$ \\
Spleen $(\mu \mathrm{mol} / \mathrm{kg})$ & $1.30 \pm 0.34$ & $0.37 \pm 0.14$ & $0.59 \pm 0.07$ \\
Colon $(\mu \mathrm{mol} / \mathrm{kg})$ & $5.49 \pm 0.80$ & $37.02 \pm 4.97$ & $0.60 \pm 0.14$ \\
Urine $($ conjugated $)(\mu \mathrm{M})$ & $194.83 \pm 29.45$ & $28.12 \pm 7.92$ & Not detected \\
Urine $($ free $)(\mu \mathrm{M})$ & Not detected & $0.14 \pm 0.04$ & Not detected
\end{tabular}

${ }^{\text {a }}$ Samples are from mice treated with AIN76 $m$ diet containing $0.05 \% \gamma$-T3 for 2 weeks. Data are shown as mean \pm S.E. $(n=5)$

in mediating the synthesis of cholesterol. In addition to HMGCR, $\delta$ - and $\gamma$-T3 also suppressed other upstream regulators of lipid homeostatic genes including DGAT2, APOB100, and SREBP1/2, leading to the suppression of triglycerides, cholesterol, and VLDL biosynthesis in HepG2 cells and enhanced the efflux of LDL through induction of LDL receptor in LDL receptor deficient mice (Zaiden et al. 2010). However, discrepancy existed in that $\delta$ - and $\gamma$-T3 only lowered serum triglycerides level, whereas total cholesterol, LDL, and HDL levels remained unchanged in a placebo-controlled human trial. Similarly, only triglyceride levels were reduced in carbon tetrachloride-induced fatty liver in rats (Yachi et al. 2010). In addition to cholesterol-related metabolism, Berbee et al. (2009) showed that $\gamma$-T3 improved post-irradiation survival and decreased radiation-induced vascular oxidative stress after a total-body irradiation. The effect of $\gamma$-T3 could be eliminated by mevalonate, the product of the reaction catalyzed by HMGCR, suggesting that the protective role of $\gamma$-T3 was through an HMGCR-dependent mechanism. In 
this study, whether the inhibition of cholesterol biosynthesis by $\mathrm{T} 3$ was involved in the process was unclear. The research from our group demonstrated that $\gamma$-T3 inhibited cancer cell growth by an HMGCR-dependent, but cholesterol independent mechanism (Yang et al. 2010).

\section{Synergistic anti-cancer actions of tocotrienols with other agents}

T3 has been shown to inhibit various cancer cell growth in many studies, generally using relatively high concentrations (above $20 \mu \mathrm{M}$ ) of T3 (Sun et al. 2008; Shah and Sylvester 2004; Takahashi and Loo 2004; Srivastava and Gupta 2006). One way to enhance the effectiveness of T3 is to use $\mathrm{T} 3$ together with other agents to synergize the anticancer activity. Several research groups reported that the combinations of $\mathrm{T} 3$ with other agents significantly potentiated the effects of T3 (Hsieh and Wu 2008; McAnally et al. 2007; Shirode and Sylvester 2010).

Our group has studied the combination of $\gamma$-T3 with atorvastatin (ATST), another agent that possesses anticancer activities, in HT29 and HCT116 colon cancer cell lines (Yang et al. 2010). We demonstrated that $\gamma$-T3 could inhibit colon cancer cell growth by MTT assays with the half-maximal inhibitory concentration $\left(\mathrm{IC}_{50}\right)$ at 30 and $17.5 \mu \mathrm{M}$, whereas the $\mathrm{IC}_{50}$ for ATST was 30 and $3.5 \mu \mathrm{M}$, respectively, for HT29 and HCT116 cells. The combination of $\gamma$-T3 with ATST synergistically inhibited colon cancer cell growth as demonstrated by isobologram analysis, with a significant decrease of the $\mathrm{IC}_{50}$ values for both agents $(9$ and $0.72 \mu \mathrm{M}$ for ATST in HT29 and HCT116 cells, respectively, and 9 and $3.6 \mu \mathrm{M}$ for $\gamma$-T3 in HT29 and HCT116 cells, respectively). $\gamma$-T3 and ATST also synergistically induced cell cycle arrest at $G_{0} / G_{1}$ phase, as determined by propidium iodide (PI) staining followed by flowcytometry and by the induction of cell cycle arrestrelated $\mathrm{p} 21^{\mathrm{Cip} / \mathrm{Waf} 1}$ level. The combination of $\gamma$-T3 and ATST also synergistically induced colon cancer cell apoptosis, as determined by Annexin V/PI staining followed by flowcytometry, by DNA fragmentation and by the cleavage of caspase 3 . Moreover, the triple combination of $\gamma$-T3, ATST, and celecoxib (CXIB, a cyclooxygenase-2 inhibitor) produced further synergistic action in HT29 cells in terms of the inhibition of cancer cell growth, the induction of cell cycle arrest, and the induction of cell apoptosis. The effect of $\gamma$-T3 was further potentiated with the $\mathrm{IC}_{50}$ decreased from $9 \mu \mathrm{M}$ in its combination with ATST, to $4 \mu \mathrm{M}$ in the triple combination in HT29 cells.

We also investigated the mechanisms of the synergistic action of $\gamma$-T3 and ATST and proposed that the synergy of $\gamma$-T3 and ATST could be attributed to their different actions on 3-hydroxy-3-methylglutaryl coenzyme A
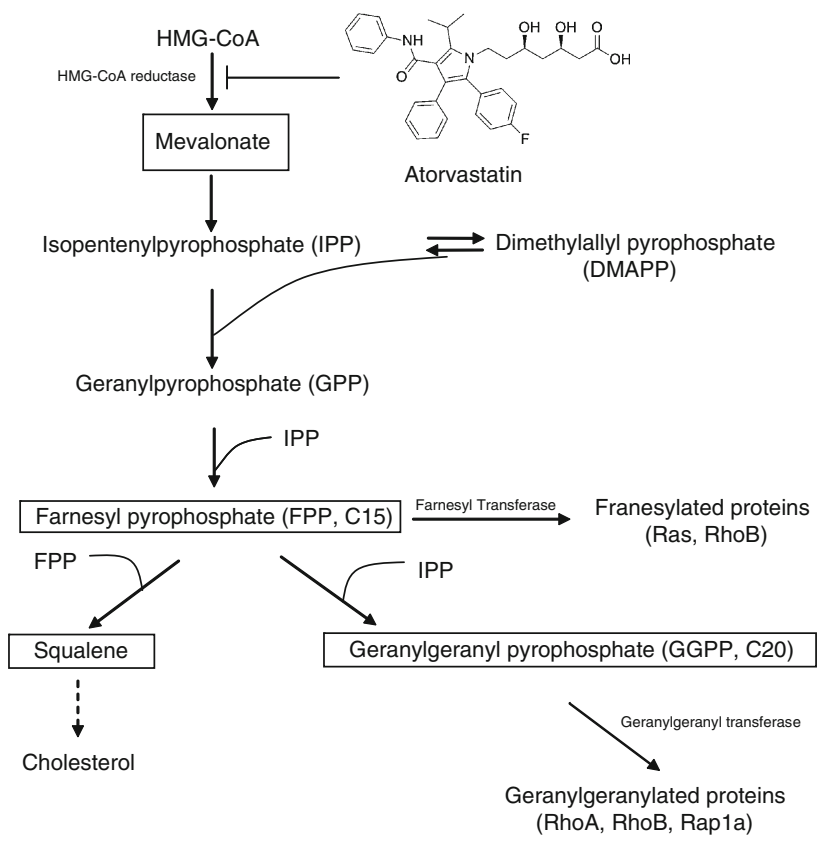

Fig. 4 Inhibition of isoprenoid biosynthetic pathway by ATST [modified from Holstein et al. (Holstein et al. 2002)]

reductase (HMGCR). ATST, as a widely prescribed cholesterol lowering drug, is an inhibitor of HMGCR. The inhibition of HMGCR activity by ATST triggered an increase of HMGCR level, possibly to compensate for the loss of enzyme activity. The addition of $\gamma$-T3 attenuated the level of HMGCR elevated by ATST (Yang et al. 2010).

The inhibition of HMGCR by $\gamma$-T3 and ATST led to the depletion of downstream metabolic products (Fig. 4). In our studies, addition of mevalonate and geranylgeranyl pyrophosphate (GGpp) eliminated the growth inhibitory and pro-apoptotic effects of ATST or its combination with $\gamma$-T3 in HT29 cells (Yang et al. 2010). However, addition of farnesyl pyrophosphate (Fpp) and squalene, two important precursors for cholesterol synthesis (Fig. 4), had no effect on the cell growth inhibition, although addition of squalene, as well as mevalonate, restored cellular cholesterol level depleted by ATST. These results suggest that, although $\gamma$-T3, in combination with ATST, has a dramatic effect on HMGCR, the rate-limiting enzyme in cholesterol biosynthesis, cholesterol did not play an essential role in the cancer cell growth inhibitory action of $\gamma$-T3 and ATST. GGpp and Fpp are involved in protein isoprenylation. Isoprenylation is essential for membrane localization and consequently affects the functions of some proteins, such as the small G-protein family. We found that depletion of GGpp by the combination of $\gamma$-T3 and ATST caused a significant decrease of membrane-bound and accumulation of cytosolic small G-proteins that undergo geranylgeranylation, such as RhoA, Rab5, and Rab6 (Yang et al. 2010). Addition of mevalonate and GGpp, but not Fpp, restored 
their level to the normal status. Interestingly, although Fpp is also responsible for protein isoprenylation and should also be depleted by the combination of $\gamma$-T3 and ATST, addition of Fpp did not restore cell growth, suggesting that protein farnesylation was not involved for cell growth inhibition induced by the combination $\gamma$-T3 and ATST. We demonstrated that the level of membrane-bound and cytosolic K-Ras, a small G-protein known to be farnesylated, was not changed upon the treatment of $\gamma$-T3 and ATST; the steady status of K-Ras abrogated the need of its farnesylation for cancer cell growth. Taken together, our studies demonstrated that $\gamma$-T3, in combination of ATST, or ATST and CXIB, synergistically induced colon cancer cell growth inhibition and the mechanism of the synergistic action of $\gamma$-T3 and ATST can be contributed to their mediation of HMGCR and the subsequent protein isoprenylation.

\section{Concluding remarks}

In addition to the most widely studied anti-cancer activities, T3s have also been reported to possess some other interesting biological activities. T3s, as members of vitamin E family, were initially studied as antioxidants. However, discrepancies still exist on whether T3s are superior to tocopherols in their antioxidant activities, and on the effectiveness ranking order among different isoforms of T3s. It is likely that the apparent antioxidant activities of tocopherols and T3s are dependent on experimental conditions (Kamat et al. 1997; Kashiwagi et al. 2009; Serbinova et al. 1991; Begum and Terao 2002; Yoshida et al. 2003). T3-rich fraction supplementation also stimulated immune response in the presence of immunogenic challenge (Radhakrishnan et al. 2009; Mahalingam et al. 2010) and exhibited potent anti-inflammatory activity by mediating inflammation-related cytokines (Wu et al. 2008). Further investigation is still needed to elucidate whether these biological functions of $\mathrm{T} 3 \mathrm{~s}$ contribute to their anti-cancer activities.

In our studies of T3s and their metabolism, we established a method to detect a complete profile of T3s together with tocopherols and their metabolites in mouse urine and fecal samples. We determined the levels of $\gamma$ - and $\delta$-T3 and $\gamma$-T3 metabolites in different mouse tissues and compared the levels T3s with those of corresponding tocopherols. These results will form the basis to elucidate the contribution of T3s and their metabolites to their anti-cancer and other biological activities in mouse studies. We also demonstrated the synergistic action of $\gamma$-T3 with ATST or ATST plus CXIB to inhibit cancer cell growth, which greatly reduced the doses needed to inhibit colon cancer cell growth as compared to each individual agent. These studies of T3s will encourage their future investigation and application in cancer prevention and therapy.

Acknowledgments This work was supported by National Institutes of Health (NIH) grants CA120915, CA133021, John L. Colaizzi Chair Endowment Fund, and Jewels of Charity award to the Cancer Institute of New Jersey and Facility Cores (supported by NIH grants ES05022 and CA72720).

\section{References}

Ahn KS, Sethi G, Krishnan K, Aggarwal BB (2007) Gammatocotrienol inhibits nuclear factor-kappaB signaling pathway through inhibition of receptor-interacting protein and TAK1 leading to suppression of antiapoptotic gene products and potentiation of apoptosis. J Biol Chem 282(1):809-820

Begum AN, Terao J (2002) Protective effect of alpha-tocotrienol against free radical-induced impairment of erythrocyte deformability. Biosci Biotechnol Biochem 66(2):398-403

Berbee M, Fu Q, Boerma M, Wang J, Kumar KS, Hauer-Jensen M (2009) Gamma-tocotrienol ameliorates intestinal radiation injury and reduces vascular oxidative stress after total-body irradiation by an HMG-CoA reductase-dependent mechanism. Radiat Res 171(5):596-605

Birringer M, Pfluger P, Kluth D, Landes N, Brigelius-Flohe R (2002) Identities and differences in the metabolism of tocotrienols and tocopherols in HepG2 cells. J Nutr 132(10):3113-3118

Birringer M, Lington D, Vertuani S, Manfredini S, Scharlau D, Glei M, Ristow M (2010) Proapoptotic effects of long-chain vitamin E metabolites in HepG2 cells are mediated by oxidative stress. Free Radic Biol Med 49(8):1315-1322

Comitato R, Nesaretnam K, Leoni G, Ambra R, Canali R, Bolli A, Marino M, Virgili F (2009) A novel mechanism of natural vitamin E tocotrienol activity: involvement of ERbeta signal transduction. Am J Physiol Endocrinol Metab 297(2):E427-E437

Comitato R, Leoni G, Canali R, Ambra R, Nesaretnam K, Virgili F (2010) Tocotrienols activity in MCF-7 breast cancer cells: involvement of ERbeta signal transduction. Mol Nutr Food Res 54(5):669-678

Fang F, Kang Z, Wong C (2010) Vitamin E tocotrienols improve insulin sensitivity through activating peroxisome proliferatoractivated receptors. Mol Nutr Food Res 54(3):345-352

Freiser H, Jiang Q (2009) Gamma-tocotrienol and gamma-tocopherol are primarily metabolized to conjugated 2-(beta-carboxyethyl)6-hydroxy-2, 7, 8-trimethylchroman and sulfated long-chain carboxychromanols in rats. J Nutr 139(5):884-889

Holstein SA, Wohlford-Lenane CL, Hohl RJ (2002) Isoprenoids influence expression of Ras and Ras-related proteins. Biochemistry 41(46): 13698-13704

Hsieh TC, Wu JM (2008) Suppression of cell proliferation and gene expression by combinatorial synergy of EGCG, resveratrol and gamma-tocotrienol in estrogen receptor-positive MCF-7 breast cancer cells. Int J Oncol 33(4):851-859

Jiang Q, Yin X, Lill MA, Danielson ML, Freiser H, Huang J (2008) Long-chain carboxychromanols, metabolites of vitamin E, are potent inhibitors of cyclooxygenases. Proc Natl Acad Sci USA 105(51):20464-20469

Kamat JP, Sarma HD, Devasagayam TP, Nesaretnam K, Basiron Y (1997) Tocotrienols from palm oil as effective inhibitors of protein oxidation and lipid peroxidation in rat liver microsomes. Mol Cell Biochem 170(1-2):131-137

Kannappan R, Yadav VR, Aggarwal BB (2010) \{Gamma\}-Tocotrienol but not $\{$ gamma $\}$-tocopherol blocks STAT3 cell signaling 
pathway through induction of protein tyrosine phosphatase SHP1 and sensitizes tumor cells to chemotherapeutic agents. J Biol Chem 285:33520-33528

Kashiwagi K, Virgona N, Harada K, Kido W, Yano Y, Ando A, Hagiwara K, Yano T (2009) A redox-silent analogue of tocotrienol acts as a potential cytotoxic agent against human mesothelioma cells. Life Sci 84(19-20):650-656

Lekli I, Ray D, Mukherjee S, Gurusamy N, Ahsan MK, Juhasz B, Bak I, Tosaki A, Gherghiceanu M, Popescu LM, Das DK (2009) Coordinated autophagy with resveratrol and gamma-tocotrienol confers synergetic cardioprotection. J Cell Mol Med 14(10): 2506-2518

Luk SU, Yap WN, Chiu YT, Lee DT, Ma S, Lee TK, Vasireddy RS, Wong YC, Ching YP, Nelson C, Yap YL, Ling MT (2010) Gamma-tocotrienol as an effective agent in targeting prostate cancer stem cell-like population. Int J Cancer 128(9):2182-2191

Mahalingam D, Radhakrishnan AK, Amom Z, Ibrahim N, Nesaretnam K (2010) Effects of supplementation with tocotrienol-rich fraction on immune response to tetanus toxoid immunization in normal healthy volunteers. Eur J Clin Nutr 65:63-69

McAnally JA, Gupta J, Sodhani S, Bravo L, Mo H (2007) Tocotrienols potentiate lovastatin-mediated growth suppression in vitro and in vivo. Exp Biol Med (Maywood) 232(4):523-531

Miyazawa T, Shibata A, Nakagawa K, Tsuzuki T (2008) Antiangiogenic function of tocotrienol. Asia Pac J Clin Nutr 17(Suppl 1):253-256

Parker RA, Pearce BC, Clark RW, Gordon DA, Wright JJ (1993) Tocotrienols regulate cholesterol production in mammalian cells by post-transcriptional suppression of 3-hydroxy-3-methylglutaryl-coenzyme A reductase. J Biol Chem 268(15):11230-11238

Radhakrishnan AK, Lee AL, Wong PF, Kaur J, Aung H, Nesaretnam K (2009) Daily supplementation of tocotrienol-rich fraction or alpha-tocopherol did not induce immunomodulatory changes in healthy human volunteers. Br J Nutr 101(6):810-815

Rickmann M, Vaquero EC, Malagelada JR, Molero X (2007) Tocotrienols induce apoptosis and autophagy in rat pancreatic stellate cells through the mitochondrial death pathway. Gastroenterology 132(7):2518-2532

Samant GV, Sylvester PW (2006) Gamma-tocotrienol inhibits ErbB3dependent PI3 K/Akt mitogenic signalling in neoplastic mammary epithelial cells. Cell Prolif 39(6):563-574

Serbinova E, Kagan V, Han D, Packer L (1991) Free radical recycling and intramembrane mobility in the antioxidant properties of alpha-tocopherol and alpha-tocotrienol. Free Radic Biol Med 10(5):263-275

Shah S, Sylvester PW (2004) Tocotrienol-induced caspase-8 activation is unrelated to death receptor apoptotic signaling in neoplastic mammary epithelial cells. Exp Biol Med (Maywood) 229(8):745-755

Shah SJ, Sylvester PW (2005a) Gamma-tocotrienol inhibits neoplastic mammary epithelial cell proliferation by decreasing Akt and nuclear factor kappaB activity. Exp Biol Med (Maywood) 230(4):235-241

Shah SJ, Sylvester PW (2005b) Tocotrienol-induced cytotoxicity is unrelated to mitochondrial stress apoptotic signaling in neoplastic mammary epithelial cells. Biochem Cell Biol 83(1):86-95

Shirode AB, Sylvester PW (2010) Synergistic anticancer effects of combined gamma-tocotrienol and celecoxib treatment are associated with suppression in Akt and NFkappaB signaling. Biomed Pharmacother 64(5):327-332

Song BL, DeBose-Boyd RA (2006) Insig-dependent ubiquitination and degradation of 3-hydroxy-3-methylglutaryl coenzyme a reductase stimulated by delta- and gamma-tocotrienols. J Biol Chem 281(35):25054-25061

Srivastava JK, Gupta S (2006) Tocotrienol-rich fraction of palm oil induces cell cycle arrest and apoptosis selectively in human prostate cancer cells. Biochem Biophys Res Commun 346(2): $447-453$

Sun W, Wang Q, Chen B, Liu J, Liu H, Xu W (2008) Gammatocotrienol-induced apoptosis in human gastric cancer SGC7901 cells is associated with a suppression in mitogen-activated protein kinase signalling. Br J Nutr 99(6):1247-1254

Takahashi K, Loo G (2004) Disruption of mitochondria during tocotrienol-induced apoptosis in MDA-MB-231 human breast cancer cells. Biochem Pharmacol 67(2):315-324

Wu SJ, Liu PL, Ng LT (2008) Tocotrienol-rich fraction of palm oil exhibits anti-inflammatory property by suppressing the expression of inflammatory mediators in human monocytic cells. Mol Nutr Food Res 52(8):921-929

Yachi R, Igarashi O, Kiyose C (2010) Protective effects of vitamin E analogs against carbon tetrachloride-induced fatty liver in rats. J Clin Biochem Nutr 47(2):148-154

Yang Z, Xiao H, Jin H, Koo PT, Tsang DJ, Yang CS (2010) Synergistic actions of atorvastatin with gamma-tocotrienol and celecoxib against human colon cancer HT29 and HCT116 cells. Int J Cancer 126(4):852-863

Yap WN, Chang PN, Han HY, Lee DT, Ling MT, Wong YC, Yap YL (2008) Gamma-tocotrienol suppresses prostate cancer cell proliferation and invasion through multiple-signalling pathways. $\mathrm{Br}$ J Cancer 99(11):1832-1841

Yoshida Y, Niki E, Noguchi N (2003) Comparative study on the action of tocopherols and tocotrienols as antioxidant: chemical and physical effects. Chem Phys Lipids 123(1):63-75

Zaiden N, Yap W, Ong S, Xu C, Teo V, Chang C, Zhang X, Nesaretnam K, Shiba S, Yap Y (2010) Gamma Delta Tocotrienols Reduce Hepatic Triglyceride Synthesis and VLDL Secretion. J Atheroscler Thromb 17(10):1019-1032

Zhao Y, Lee MJ, Cheung C, Ju JH, Chen YK, Liu B, Hu LQ, Yang CS (2010) Analysis of multiple metabolites of tocopherols and tocotrienols in mice and humans. J Agric Food Chem 58(8):4844-4852 